Session 2251

\title{
A Multimedia Based Laboratory Course for Environmental Engineering
}

\author{
Lynn E. Katz ${ }^{1}$, Lenly J. Weathers ${ }^{2}$, Ronald Kozlowski ${ }^{2}$, Mike Scott ${ }^{2}$ and Will \\ Manion $^{2}$ \\ ${ }^{1}$ University of Texas at Austin $/{ }^{2}$ University of Maine
}

\begin{abstract}
$\underline{\text { Abstract }}$
The focus of this project is on the development of interactive multi-media laboratory modules to accompany a physical laboratory course in environmental engineering process dynamics. The courseware modules are intended to enhance the laboratory experience by ensuring that students have a firm grasp of fundamental concepts and experimental methods for conducting process engineering laboratories.
\end{abstract}

Three major topics are covered in the laboratory course: reactor theory, kinetics, and mass transfer. The goal of the project is to produce one to two multi-media modules for each major topic. The modules are designed to teach students how to collect, analyze and interpret data to obtain design parameters for engineered processes or mathematical modeling of contaminant transport in the environment. Each module contains concept, laboratory and analysis tutorials, videos of each lab, and an animated laboratory that can be used by the students to evaluate the effect of different design and operational parameters.

Students are expected to review the modules at various points in the learning process. Prior to conducting the physical laboratory, students are expected to complete the concept and pre-lab tutorials, view the laboratory videos, and "run" the virtual laboratory using the operating parameters that will be employed in the physical laboratory. The objective of this assignment is to allow students to acquire visual and conceptual familiarity with the experiment and to determine sampling times and data requirements. After they have collected their own data in the laboratory, they will use the courseware again to learn data analysis techniques and to observe the impact of varying parameter conditions. The expected benefits of these modules include improving the quality of data obtained in the laboratory, enhancing student understanding of key concepts, and allowing them to establish the link between theory and application.

Introduction

The objective of this work was to develop a course for undergraduate civil engineers that provides an introduction to the fundamentals of environmental processes which control contaminant transport in the environment. More specifically the course is designed to emphasize basic process fundamentals, provide exposure to design techniques and allow students to acquire familiarity with the procedures for obtaining and determining design parameters for full scale systems from laboratory testing. The course is divided into three major sections: environmental 
process flow dynamics, chemical reaction kinetics, and partitioning of contaminants between air/water, soil/water and organic/water systems. Concepts covered in these topics are necessary for obtaining an understanding of all biological and physicochemical process operations and, therefore, this course is a logical precursor to courses in environmental science and engineering, geology and chemical engineering.

The course presents environmental engineering principles in the classroom (two fifty minute class lectures/week ) and demonstrates the application of these principles in a weekly laboratory. One of the key features of the course was the introduction of the laboratory component which provides visualizations of the concepts developed in classes to help students understand how macroscopic data is used to describe microscopic environmental processes. A laboratory component devoted to each major topic demonstrates how parameters used to describe contaminant transport are estimated in the laboratory and used in the field.

The first session of each laboratory is devoted to analytical method development for the particular compound to be tested or treated in the laboratory. In this session the instrumental method of analysis is presented and described, the principle of operation is outlined, and the students learn to use the equipment, determine the detection limits for the analyte and prepare calibration curves. In the second session of each laboratory, the students collect data in bench scale reactors to estimate reaction parameters that could be used in a field-scale reactor. In the final week of each section the students work in a multi-media classroom to analyze the data. This final session was added to the laboratory because our previous experience with laboratory classes has shown that students generally spend a disproportionate amount of time trying to understand how to analyze data rather than understanding its significance. We have found that it is more productive and efficient for the students to analyze the data in a guided, active learning environment. With this approach, the major portion of the laboratory report preparation is spent evaluating and interpreting the significance of the data, rather than trying to interpret data which is confounded by errors in the data analysis.

While the addition of this final laboratory session may have reduced the amount of time and frustration associated with the data analysis, it did not address many of the other shortcomings that are associated with classroom laboratory experiences. These shortcomings include the poor quality and quantity of data collected in the laboratory and the students' incomplete understanding of the effects of various design parameters on the processes studied. One of the potential reasons for the poor quality and quantity of data collected by the students is that the students often begin the laboratory with very little familiarity with the process equipment or the sampling procedures. The major reason that they do not obtain a good understanding of the effects of changing the design parameters is that they do not have an opportunity to run multiple experiments in the laboratory due to a lack of time and/or resources.

The objective of this work is to develop multimedia laboratories that address these issues by providing students with: 1) an animated laboratory that enables experiments to be conducted numerous times under different operating conditions to evaluate the effect of design parameters; and, 2) videos of the laboratory that the students can view prior to entering the laboratory in order to acquire some familiarity with the equipment and procedures to be used. It is important to point out that the animated laboratories are intended to accompany a hands-on laboratories and not to replace them. It is our hypothesis that there is significant potential for gaining better 
understanding of the processes using the combined approach because it is possible to experience the laboratory first hand and "run" the simulated laboratory numerous times under different scenarios and parameter conditions. This provides students with an opportunity to observe a laboratory, develop a more sound understanding of the fundamental aspects of the operative processes, and then to revisit the laboratory to better understand the impact of different parameters on the process efficiency.

\section{Courseware Components}

Three major topics are covered in the laboratory course: reactor theory, kinetics, and mass transfer. The goal of the project is to produce one to two multi-media modules for each major topic as shown in Table 1 below. Many of the laboratories listed in Table 1 are published experiments that are routinely conducted in environmental engineering courses using relatively similar conditions. ${ }^{1,2}$

Table 1. Laboratory Modules

\begin{tabular}{|l|l||}
\hline Major Topic & Laboratory \\
\hline Reactor Theory & $\begin{array}{l}\text { Ideal Reactor Tracer Study } \\
\text { Non-Ideal Reactor Tracer Study }\end{array}$ \\
\hline Kinetics & $\begin{array}{l}\text { Kinetics of Iron Oxidation } \\
\text { Kinetics of Biodegradation }\end{array}$ \\
\hline Mass Transfer & Stream Reaeration \\
\hline
\end{tabular}

The software will be designed to teach students how to collect and analyze data to obtain design parameters for engineered processes or mathematical modeling of contaminant transport in the environment. The courseware will include videos or animations of the laboratory component of the course, interactive tutorials to introduce theoretical concepts and demonstrate data analysis techniques, and simulations of field applications of the laboratory results. Specifically, each module contains:

1. an interactive tutorial describing the conceptual theories associated with the experiment and demonstrating examples of the processes in natural and engineered systems;

2. an interactive pre-lab tutorial describing the specific reactions or experiments to be conducted;

3. annotated video clips of the physical laboratory illustrating how to conduct experiments to obtain data required as part of the design procedure for field-scale processes;

4. a digital simulation production that consists of an animation of the experiment that is enhanced to allow students to "collect" effluent data at any point in time by clicking on a sampling port; 
5. an interactive tutorial demonstrating how design parameters are obtained from the laboratory data and allowing students to gauge their understanding of the material; and,

6. an annotated spreadsheet that is linked to the data collection animation to assist students in determining design parameters for field applications.

The courseware is being developed using a variety of tools including Dreamweaver and Authorware (Macromedia, Inc.) for the tutorials and Director and Shockwave (Macromedia, Inc.) for the animated laboratories. Authorware is an icon-based multimedia authoring environment ideally suited for interactive courseware and student assessment. Director is one of the foremost multimedia authoring tools on the market today. In conjunction with its programming language Lingo, its ability to create Shockwave movies allows us to create powerful multimedia simulations on the web. A brief description of the major components of the courseware is provided below.

Animated Laboratories. The development of the digital simulation productions for each laboratory can be broken down into four parts: the interface, the interactivity, the calculations, and the output. Each interface is a digital image that emulates a real laboratory in detail and complexity, is user-friendly, and integrates well with the rest of the software. A prototype of one system in which the laboratory for studying the kinetics of iron oxidation is shown in Figure 1.

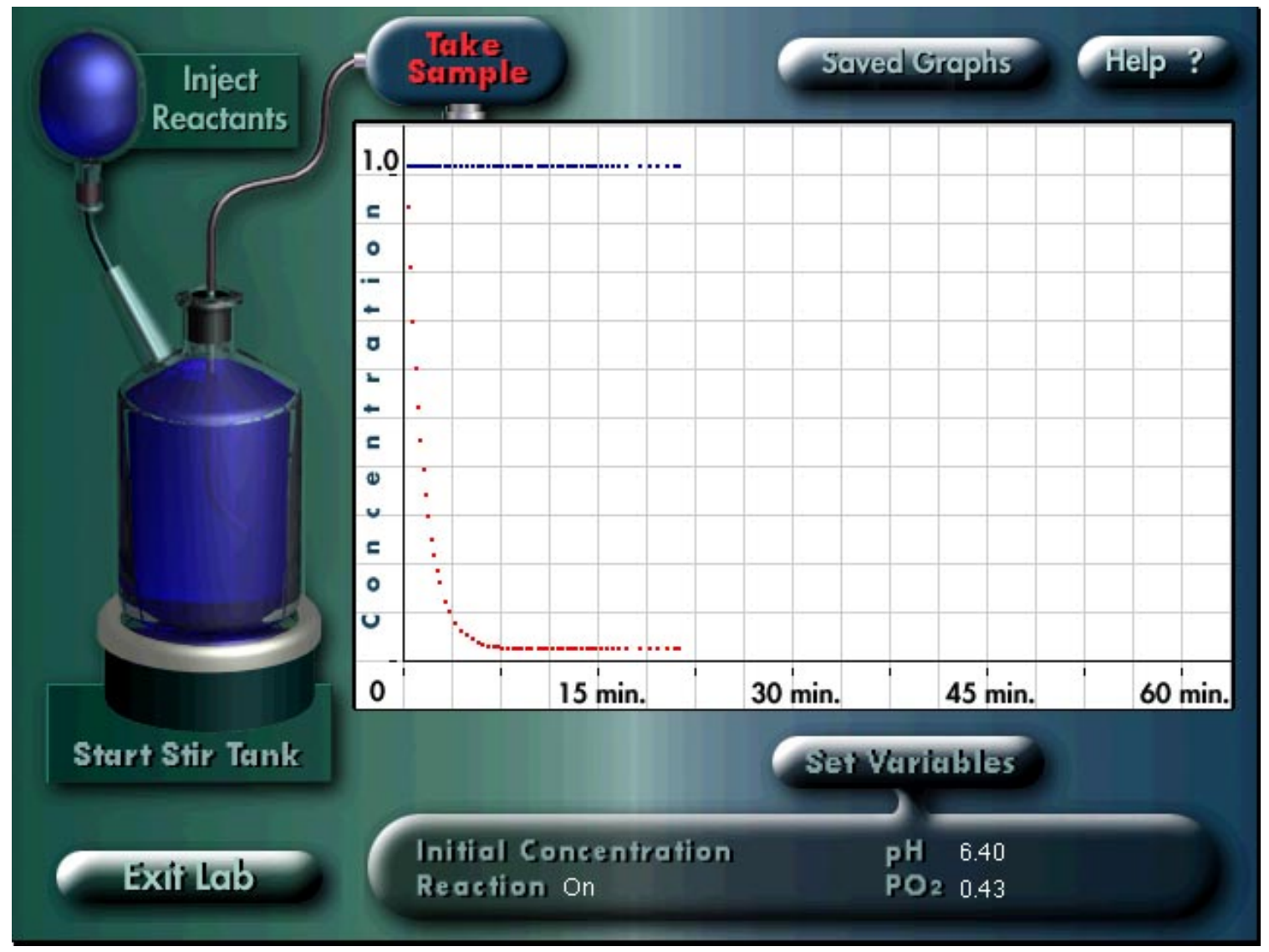

Figure 1. Animation for the Kinetics of Iron Oxidation Laboratory 
The interactivity is defined by how users conduct the experiments, how they change the settings for the experiment and how that information is input. For example, in the prototype shown in Figure 1 the user defines the parameters for operating the experiment by clicking on the Set Variables button and a new interface with sliding scales allows the user to set each parameter value. To start the system, the user clicks on the Start Stir Tank button and the tank fills and sets the operating conditions such as $\mathrm{pH}$ and temperature to the selected values. In addition, the time scale on the abscissa (e.g. minutes, hours, days or years) is determined based on the time it takes for greater than $99.99 \%$ reduction in the reactive component concentration. Once the reactor is prepared, the user initiates the experiment by clicking on the Inject Reactants button. The user is warned that sampling should start when the reactants enter the reactor.

The injection process is visually apparent by the change in color in the tank. Two colors of reactants are available, a blue conservative tracer and a red reactive component. The color in the reactor is determined by the blending of the two colors. For the batch experiment shown in Figure 1, the color of the reactor initially becomes purple upon injection indicating that the conservative tracer and the reactive component are present at maximum concentration. From the settings the user selected for the experiment, a series of calculations are performed using analytical or numerical solutions to the theoretical equations to simulate the experiment that would be performed using the input conditions. Over time the concentration of the reactive component decreases and the red color fades whereas the conservative tracer remains at the same level of intensity. Thus, the reactor turns blue with time. During this time, the user clicks on the sample button to simulate sample collection and data points for the conservative and reactive component are calculated and placed on the graph. In addition the data are simultaneously saved in an ASCII file that can be imported into a spreadsheet environment such as Microsoft EXCEL. Other features of the animated laboratories include on-line help and the ability to graph and compare multiple data sets derived using different parameter settings.

Tutorials. The tutorials are required to be fully integrated with the digital simulation production. Each module has two introductory tutorials that introduce the student to the concepts applied in the laboratory. The tutorials are designed to track student responses to questions for evaluation purposes, allow students three attempts to answer questions posed in the tutorial before providing correct answers and will fully involve the students in an interactive learning experience that prepares them to conduct the laboratory. The first of these tutorials reviews general concepts for the laboratory. If the students feel that they have a good understanding of the concepts prior to reviewing the concept tutorial, they can take an optional pre-test to demonstrate their understanding. If the user answers more than seventy percent of the questions correctly, they are allowed to proceed to a tutorial that applies the concepts to the specific laboratory experiment to be conducted.

For example, in the case of the kinetics of iron oxidation module the concept tutorial covers material such as rate expressions, reaction orders and reaction stoichiometry. The pre-lab tutorial applies these concepts to develop the rate expression for iron oxidation and provides an overview that describes how data can be collected to determine rate constants for the batch system. 
After the students collect data from the digital simulation they will learn to analyze the data in a final tutorial that takes them through the data analysis using Microsoft EXCEL (or other suitable data analysis tool). This tutorial will be designed to instruct the student as to the proper procedures for reducing the raw data collected in the laboratory and extracting design parameters such as reaction rates, non-ideal flow parameters, or mass transfer coefficients. It will also explain how these parameters are then used to design full scale systems.

The data from each digital simulation will be saved in ASCII files that will be imported to annotated Microsoft EXCEL Spreadsheets that are designed to assist the students in the data reduction process. For example, these spreadsheets contain notes in particular cells that explain the calculation that is to be performed.

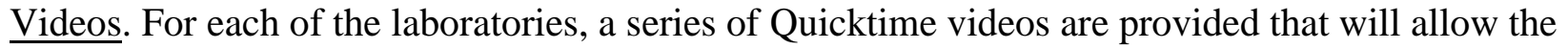
students to view the laboratory prior to conducting their experiments. The videos are accompanied by an audio presentation that explains each of the steps required to set up, collect, and analyze the samples. Thus, the goal of the videos is to allow the students to acquire some familiarity with the process equipment and experimental procedures in a visual mode.

\section{$\underline{\text { Dissemination }}$}

All of the modules will be available on the web. The tutorials are being designed using Dreamweaver (Macromedia, Inc.), a professional authoring tool for creating web pages. Dreamweaver uses Roundtrip HTML ${ }^{\mathrm{TM}}$ technology along with the ability to use DHTML. Along with DeamWeaver we are using Flash 2, (Macromedia, Inc.) that allows for effective web animation due to its small bandwidth requirements. We will be using Authorware (Macromedia, Inc.) to track student progress throughout the multi-media laboratory modules. The animated experiments are currently being designed in Macromedia Director. Using Director's programming language, Lingo, we were able to apply analytical solutions to simulate the laboratory data under a range of conditions. One of the important features of Director is its ability to convert the program into Shockwave. Using Macormedia's Shockwave plug-in enables Director movies to run on the Web. However, we have also been successful in streaming these experiments off of the web.

\section{Summary}

The multimedia courseware being developed for courses in environmental engineering process dynamics is designed to supplement a hands-on laboratory in order to improve the quality of data obtained in the laboratory and enhance student understanding of key concepts and the effect of varying operating and design parameters. The modules will undergo evaluation by faculty and students at a number of institutions that offer similar courses in their programs. Further evaluation and dissemination will be available through the web.

\section{$\underline{\text { Acknowledgement }}$}

This work has been supported by the National Science Foundation, Grant Nos. DUE-9653149 and BES-9625047. 


\section{$\underline{\text { References }}$}

1. AEEP (Association of Environmental Engineering Professors) "Environmental Engineering: Unit Operations and Unit Processes - Laboratory Manual," Fourth Edition, 1988.

2. Jenkins, D.V., Snoeyink, V.L., Ferguson, J.F., and Leckie, J.O., "Laboratory Manual: Water Chemistry," Third Edition, AEEP, John Wiley and Sons, New York, 1980.

Lynn E. Katz received her B.S.E in Environmental Engineering from Johns Hopkins University in 1980. She then worked as a design engineer in an environmental engineering consulting firm for two years. She earned M.S. degrees in Environmental Engineering and Chemistry and a Ph.D. in Environmental Engineering at the University of Michigan. In addition, she was employed as a laboratory manager for the Department of Civil and Environmental Engineering at the University of Michigan for several years. She joined the faculty in the Department of Civil and Environmental Engineering at the University of Maine in 1991 and in 1998 she joined the faculty in the Department of Civil Engineering at the University of Texas at Austin. Her research addresses the fate and transport of organic and inorganic contaminants in natural and engineered systems and focuses on reactions at surface/water interfaces.

Lenly J. Weathers is a graduate of The University of Missouri (BSME,MSME), Texas A\&M University (MSCE) and The University of Iowa (Ph.D.,Civil and Environmental Engineering). Prior to receiving his Ph.D., heworked in hazardous waste site remediation. Dr. Weathers has been withThe University of Maine since 1996. His research focuses on the remediation of contaminated groundwater using combined metalliciron/microbial treatment systems and biodegradation of xenobioticcompounds such as pesticides, chlorinated solvents and petroleumhydrocarbons under anaerobic and aerobic conditions.

Mike Scott received his BS in Surveying Engineering at the University of Maine in 1989. Since then he has been employed by Computing Instructional Technologies at the University of Maine where he developed and implemented campus wide Email and computer conferencesystem where he has designed and implemented computer based classrooms and multimedia lecture halls and routinely works with faculty to integrate technologiesinto their teaching environment. In 1989 he founded ASAP Media Service a student based multimedia design group at the University of Maine involved in the exploration, understanding and implementation of the integration of technology and communication.

Ronald N. Kozlowski received a Bachelor of Science degree at the University of Maine at Orono in $1997 \mathrm{He}$ is currently working towards his Masters in Developmental Biology at the University of Maine. This involves the production of two CD-ROMs, FlyCycle CD and an Interactive Developmental Biology Lab Book . Ron is also an employee of ASAP Media Services, a student run multimedia group at the University of Maine. Where he is currently working with Lynn Katz to develop A Multimedia Based Laboratory Course for Environmental Engineering.

Will Manion graduated with a B.S. in Engineering: Wood Products, Construction and Management from The State University of New York atSyracuse in 1989. He obtained an M.S. in Civil and EnvironmentalEngineering from The University of Maine in 1992. Mr. Manion has beenemployed by the University of Maine since 1992. He manages the civiland environmental engineering laboratories, as well as being active ingeotechnical and structural research. Will currently teaches CIE 366Soils Laboratory, coordinates CIE 111 Materials Laboratory, and has anactive part in web development for the department. 